\title{
The left ventricular dysfunction questionnaire (LVD-36): reliability, validity, and responsiveness
}

\author{
C J O’Leary, P W Jones
}

\begin{abstract}
Objective-To examine the reliability, validity, and responsiveness of a new health status measure (LVD-36) for patients with left ventricular dysfunction which was designed with emphasis on content validity, clarity, brevity, and ease of use.

Design-At baseline, patients completed the LVD-36 and a range of measures reflecting general health and disease severity. The LVD-36 was repeated after one week. After six months, it was repeated again, along with a transition question to measure global changes in health.

Setting-Patients were recruited from the cardiology and general medical clinics at a south west London hospital.

Patients-60 patients with chronic left ventricular dysfunction.

Interventions-None.

Main outcome measures-Short form 36 questionnaire (SF-36), Minnesota living with heart failure questionnaire (LIhFE), New York Heart Association criteria, and exercise performance and echocardiographic tests.

Results-The LVD-36 showed good internal consistency $(\kappa=0.95)$ and repeatability $\left(r_{\mathrm{i}}=0.95\right)$. Its scores were significantly associated with SF-36 mental and physical component scores $(r=-0.48$ and $-0.75 ; \mathrm{p}<0.0001)$, with exercise capacity $(r=-0.52 ; \mathrm{p}<0.0001)$, and with systolic shortening fraction $(r=-0.27$; $<<0.05)$. Change in the LVD-36 over six months was associated with change in overall health $(\mathrm{F}=5.7 ; \mathrm{p}<0.001)$. In tests of validity and responsiveness, the LVD-36 performed similarly to or marginally better than the LIhFE.

Conclusions-The LVD-36 showed a high level of reliability and validity, and appears to measure changes in health. It provides a short, simple, valid, and reliable measure of health status in patients with left ventricular dysfunction.
\end{abstract}

(Heart 2000;83:634-640)

Keywords: health status; quality of life; heart failure; left ventricular dysfunction

In patients with chronic diseases, such as congestive cardiac failure, measurement of health status ("quality of life") is becoming an important method of assessing the impact of the disease and the efficacy of treatment. Three health status questionnaires have been developed for patients with congestive heart failure..$^{1-3}$ These vary in development process, content, length, and complexity of application.

The chronic heart failure questionnaire $(\mathrm{CHFQ})^{1}$ was developed by presenting a pool of 123 items to 88 patients, who rated their importance on a five point scale. Item selection was based on frequency and importance ratings to ensure that items were relevant to the population. This method of item selection includes only those items experienced by the majority of the population, and thus may reduce the ability of the questionnaire to detect differences in health. In that study, the sample used was not equally balanced in terms of sex (62 male/16 female), and the choice of items is thus more likely to reflect the preferences of male patients and to produce a sex bias within the questionnaire. A section of the CHFQ is individualised, patients being asked to nominate those activities associated with shortness of breath that affected them most often and most importantly. This approach gets closer to impaired quality of life for the individual patient than questionnaires that are completely standardised and which thus treat all patients as though they are "typical" or "average." The method of questionnaire administration involves a trained administrator and is rather time consuming.

Items used in the Minnesota living with heart failure questionnaire ${ }^{2}$ (LIhFE) were drawn from those in a general health questionnaire, the sickness impact profile (SIP). Patients with congestive heart failure selected 21 items from that questionnaire which they experienced and attributed to their disease. These items formed the LIhFE. This method of item selection is entirely dependent upon the presence of suitable items in an existing general health questionnaire. Thus, while the LIhFE is made up of items that are appropriate to heart failure patients, it does not necessarily include all relevant items, but merely the best of those that were already available. Important effects of heart failure may have been missed because no attempt was made to identify them. It should be noted also that the SIP has been shown to be insensitive to changes in health in other populations. ${ }^{4}$ A study by Rector and colleagues used the LIhFE to compare the health status of 804 patients randomly assigned to receive either enalapril or a combination of hydralazine and isosorbide dinitrate. ${ }^{5}$ There were no differences in health status between the two groups at baseline or at follow up. After three months of treatment, both groups showed a slight improvement in health status, which then worsened throughout the length of the trial. This pattern was also reflected in the physiological variables 
used. No placebo group was used, so it is unclear whether the rate of decline was reduced for these patients. The investigators suggest that health status may not have been measured as accurately as possible and that "some improvement in physical ability might not have been addressed by the questions that were asked."

A third questionnaire was developed in Sweden specifically for patients with severe heart failure. ${ }^{3}$ This has been translated into an English version, but the translation does not appear to be particularly good as it contains such questions as: "Did you feel well at ease during the last week?". This raises doubts about the translation process, and also concern as to whether meanings originally intended by the developers were correctly conveyed into the English version.

To overcome some of the problems with the existing questionnaires, a new instrument - the left ventricular dysfunction questionnaire (LVD-36) - has been developed specifically for patients with chronic left ventricular dysfunction. It was designed to measure the impact of left ventricular dysfunction on daily life and

Table 1 The left ventricular dysfunction questionnaire (LVD-36)

Please answer the following questions as you are feeling these days. Tick either true or false for each question.

\begin{tabular}{|c|c|c|}
\hline Because of my heart condition: & True & False \\
\hline I suffer with tired legs & $\square$ & $\square$ \\
\hline I suffer with nausea (feeling sick) & 口 & 口 \\
\hline I suffer with swollen legs & $\square$ & $\square$ \\
\hline Because of my heart condition: & True & False \\
\hline I am afraid that if I go out I will be short of breath & $\square$ & $\square$ \\
\hline I am frightened to do too much in case I become short of breath & 口 & 口 \\
\hline I get out of breath with the least physical exercise & $\square$ & 口 \\
\hline I am frightened to push myself too far & $\square$ & $\square$ \\
\hline I take a long time to get washed or dressed & $\square$ & $\square$ \\
\hline \multicolumn{3}{|l|}{$\begin{array}{l}\text { If you do not do these activities for any reason other than your heart } \\
\text { condition, then please tick false }\end{array}$} \\
\hline Because of my heart condition: & True & False \\
\hline I have difficulty running, such as for a bus & $\square$ & 口 \\
\hline I have difficulty either jogging, exercising or dancing & $\square$ & $\square$ \\
\hline I have difficulty playing with children/grandchildren & $\square$ & $\square$ \\
\hline I have difficulty either mowing the lawn or hoovering/vacuum cleaning & $\square$ & $\square$ \\
\hline Because of my heart condition: & True & False \\
\hline I feel exhausted & $\square$ & 口 \\
\hline I feel low in energy & $\square$ & 口 \\
\hline I feel sleepy or drowsy & $\square$ & 口 \\
\hline I need to rest more & $\square$ & $\square$ \\
\hline I feel that everything is an effort & 口 & 口 \\
\hline My muscles feel weak & $\square$ & $\square$ \\
\hline I get cold easily & 口 & 口 \\
\hline I wake up frequently during the night & $\square$ & $\square$ \\
\hline I have become frail or an invalid & $\square$ & $\square$ \\
\hline Because of my heart condition: & True & False \\
\hline I feel frustrated & $\square$ & $\square$ \\
\hline I feel nervous & $\square$ & 口 \\
\hline I feel irritable & $\square$ & $\square$ \\
\hline I feel restless & 口 & 口 \\
\hline I feel out of control of my life & $\square$ & $\square$ \\
\hline I feel that I can not enjoy a full life & 口 & 口 \\
\hline I've lost confidence in myself & $\square$ & $\square$ \\
\hline Because of my heart condition: & True & False \\
\hline I have difficulty having a regular social life & $\square$ & $\square$ \\
\hline There are places I would like to go to but can't & 口 & 口 \\
\hline I worry that going on holiday could make my heart condition worse & 口 & $\square$ \\
\hline I have had to alter my lifestyle & 口 & 口 \\
\hline I am restricted in fulfilling my family duties & $\square$ & 口 \\
\hline \multirow{2}{*}{ I feel dependent on others } & $\square$ & $\square$ \\
\hline & True & False \\
\hline I find it a real nuisance having to take tablets for my heart condition & $\square$ & $\square$ \\
\hline My heart condition stops me doing things that I would like to do & $\square$ & $\square$ \\
\hline
\end{tabular}

PLEASE CHECK THAT YOU HAVE ANSWERED ALL THE QUESTIONS THANK YOU FOR YOUR TIME wellbeing. Its development process used a hierarchical item selection procedure $e^{6-8}$ to create a short questionnaire that is simple to complete and score, yet retains the necessary psychometric requirements of a health status measure. This paper is concerned with tests of the validity, reliability, and responsiveness of LVD-36 and provides a comparison of its performance with that of the LIhFE.

\section{Methods}

All patients participating in this study gave written informed consent. The study was approved by the Wandsworth District Health Authority ethics committee.

DEVELOPMENT OF THE LVD-36: A BRIEF

DESCRIPTION

Full details of the development of the LVD-36 are given elsewhere. ${ }^{89} \mathrm{~A}$ pool of 179 items was created using information from a review of published reports, existing questionnaires, semi-structured interviews with patients with left ventricular dysfunction, and consultation with clinicians. The items were presented to 139 patients with left ventricular dysfunction who were asked to respond to each item as true or false (as it applied to them), along with two global questions concerning health and impairment of function. Items were then removed from the pool if they were not associated with either of the global questions, if they were endorsed by the majority of the sample, if they were associated with sex, age, or disease duration, or if the endorsement rate was low and the association with global health was significant but weak. ${ }^{9}$ A principal components analysis was carried out on the remaining items. Those items that loaded highly on the first component and weakly on the subsequent components were retained to form the questionnaire.

\section{QUESTIONNAIRES USED}

\section{LVD-36}

The LVD-36 is a 36 item questionnaire for patients with left ventricular dysfunction (table 1). Responses are dichotomous (true or false). True responses are summed and the sum is expressed as a percentage, so that 100 is the worse possible score and 0 the best possible score. The LVD-36 takes approximately five minutes to complete.

\section{Medical outcomes study, SF-36}

The short form 36 questionnaire (SF-36) was used to measure general health status. It contains 36 discrete items that produce eight component scores and two overall summary scores. Scores run from 0 (worse possible score) to 100 (best possible score). The SF-36 takes approximately 15 minutes to complete.

\section{LIhFE}

The LIhFE is a condition specific questionnaire for patients with heart failure. It consists of 21 items, each of which is scored on a six point scale $(0-5)$. The score runs from 0 (best possible score) to 105 (worse possible score). To aid comparisons with the other questionnaires, the LIhFE scores were expressed as a 
percentage of the maximum possible score. The LIhFE may be self completed or read aloud to respondents. It takes approximately five minutes to complete.

\section{ENTRY CRITERIA}

Patients were recruited from the dilated cardiomyopathy, heart failure, and general medical clinics at St George's Hospital. They were recruited to the study if they fulfilled the following criteria:

- A diagnosis of chronic left ventricular dysfunction, left ventricular heart failure, congestive heart failure, or dilated cardiomyopathy.

- An echocardiogram within the previous six months which indicated an abnormally functioning or dilated left ventricle. This included any of the following:

(i) ejection fraction $\leqslant 50 \%$;

(ii) systolic shortening fraction $\leqslant 29 \%$;

(iii) left ventricular cavity in diastole $>$ normal range ${ }^{10}$;

(iv) left ventricular cavity in diastole $>$ normal range ${ }^{10}$.

- No myocardial infarct in the previous year.

- No coexisting or unrelated condition that might impair exercise capacity.

\section{STUDY PROTOCOL}

Baseline

Patients fulfilling the entry criteria were asked to complete the SF-36, LIhFE, and the LVD-36. Sixty seven patients were approached and asked to take part in the study, of whom five refused. The remaining patients either completed the questionnaires while at the clinic, or in five cases agreed to take the questionnaires home and return them the following day. Of these latter, three returned the questionnaires by post the following day. In all, 60 patients completed baseline questionnaires. The questionnaires were presented in random order. After completing the LVD-36, the patients were asked if their condition affected them in another important way that was not addressed by this questionnaire.

Fifty seven patients had an $M$ mode and cross sectional transthoracic Doppler echocardiogram on the day they completed the questionnaire. The remaining three patients had an echocardiogram within three months of completion of the questionnaires. Left ventricular dimensions in diastole and systole, ejection fraction, and systolic shortening fraction were recorded. Thirty eight patients also had recent treadmill exercise tests, performed to Bruce protocol, as a part of their routine clinical assessment. Of these, 18 had exercise tests on the day the questionnaire was completed. For the remaining 20 patients, the mean (SD) time between exercise test and questionnaire completion was 10.6 (9.1) months. Achieved maximum oxygen uptake ( $\left.\mathrm{Vo}_{2} \mathrm{max}\right)$ and duration of exercise were recorded. Patients were also rated on the New York Heart Association (NYHA) functional classification scale. ${ }^{11}$
Test of short term repeatability

Fifty two patients were telephoned after approximately one week (range one to three) and asked to complete the LVD-36 over the telephone. Each call took approximately five minutes. Two additional patients were sent the LVD-36 and a stamped addressed envelope. Of these, one patient returned the questionnaire. In all, 53 patients were contacted after one week to complete the LVD-36. This approach to test-retest reliability was required because of the distance that many of the patients had to travel. At worst, the effects of different modes of administration would have led to a reduction in the apparent repeatability of the questionnaire (that is, provided a conservative estimate of it reliability).

\section{Follow up}

After approximately six months, patients were contacted to complete the LVD-36, the LIhFE, and a global change question. The preferred mode of administration was for the researcher to read the questionnaires aloud over the telephone. The questionnaires were read aloud exactly as written, including all instructions. Care was taken not to introduce any bias and not to answer any question on the behalf of the patient.

Seven patients could not be contacted by telephone and were sent the questionnaires, along with a stamped addressed envelope. Of these, five returned the questionnaires. Ten patients asked to complete the questionnaires while at hospital for their six month follow up appointment instead of over the telephone. One patient died during the six month period. In all, 49 patients completed the three questionnaires.

\section{ANALYSIS}

Repeatability

The LVD-36 was presented to patients twice, one to three weeks apart. An intraclass correlation coefficient between baseline and repeat questionnaire scores was calculated.

\section{Internal consistency}

The internal consistency of baseline scores was calculated using the Kuder-Richardson coefficient. This is the statistical equivalent to Cronbach's $\alpha$ for dichotomous data.

Validity

To validate the LVD-36, cross sectional comparisons were made between it and a number of established measures. These were selected to reflect the range of effects of the disease. The NYHA scale was used as a measure of disease severity. The echocardiogram was used as a measure of left ventricular dysfunction. The SF-36 was used as a reference measure of general physical and mental impairment. The treadmill exercise test was used as a measure of physical impairment. The underlying prediction was that the LVD-36 would be positively correlated with worsening disease state. In addition, the validity of the LVD-36 was tested further by comparing it with the LIhFE-an 
existing health status measure developed specifically for heart failure.

\section{Responsiveness}

The responsiveness of both the LVD-36 and the LIhFE was assessed by administering them again after six months, together with a transition question (a global question asking about change since the last visit). The transition question had a seven point response scale. Changes in LVD-36 and LIhFE scores were calculated by subtracting the score at six months from that at baseline, so that a positive change indicated improvement. The association between change in LVD-36 score and the rating on the transition question was calculated using analysis of variance (ANOVA). The effect size was calculated by dividing mean change with the standard deviation of baseline.

For all analyses, significance was accepted at $\mathrm{p}<0.05$. Parametric and distribution independent tests were performed throughout the analyses. No major discrepancies between these two approaches were found. The results presented are from the parametric tests to allow easier presentation of the size of the association between variables.

\section{Results}

PATIENT DETAILS

Data from 60 patients ( 46 male and 14 female) were available. Their mean (SD) age was 60 (13.3) years, left ventricular cavity diastolic dimension $6.1(0.7) \mathrm{cm}$, systolic dimension 4.7 $(0.8) \mathrm{cm}$, ejection fraction $45.4(11.8) \%$, and systolic shortening fraction $22.1(7.1) \%$. The causes of left ventricular dysfunction were: 43 with dilated cardiomyopathy, 10 with ischaemic heart disease, one with diabetes, two with hypertension, three with alcohol induced dilated cardiomyopathy, and one with multiple aetiologies. There were 30 patients in NYHA functional class I, 17 in class II, 11 in class III, and two in class IV. There were no patients in class V.

BASELINE LVD-36 AND LIhFE SCORES

Sixty patients completed the LVD-36, with no missing data. The mean score was 39.0 (28.9). The minimum LVD-36 score was 0 and the maximum was 94.4 .

Fifty four patients completed the LIhFE, with no missing data. The mean score was 29.7 (22.7). The minimum LIhFE score was 0 and the maximum was 87.6.

The distributions of LVD-36 and LIhFE scores are shown in fig 1 . In comparison with the LIhFE scores, the distribution of the LVD-36 scores appeared to be more evenly spread across the scoring range.

FLOOR AND CEILING EFFECTS

Floor and ceiling effects were calculated using the percentage of patients achieving the worst score (floor effect) and the percentage achieving the best score (ceiling effect). For the LVD-36, the floor effect was $0 \%$ and the ceiling effect was 3\%-that is, no patients achieved the worst possible score and only 3\% achieved the best possible score. For the LIhFE, the floor effect was $0 \%$ and the ceiling effect was $4 \%$.

RELATION WITH AGE, SEX, AND AETIOLOGY

There was no association between the LVD-36 and age (Pearson's $r=0.04 ; \mathrm{p}=0.76$ ), sex $(t=-1.7 ; \mathrm{p}=0.10)$, or aetiology $(\mathrm{F}=0.72$; $\mathrm{p}=0.61)$.

The LIhFE was analysed in the same way. The results were similar: there was no significant association between LihFE and age $(r=0.05 ; \mathrm{p}=0.72)$, sex $(t=-1.6 ; \mathrm{p}=0.11)$, or aetiology $(\mathrm{F}=0.88 ; \mathrm{p}=0.50)$.

\section{RELIABILITY}

The repeatability of the LVD-36 was high over one week. The intraclass correlation $\left(r_{\mathrm{i}}\right)$ was 0.95 . The repeatability of the LihFE was not tested.

The internal consistency of the LVD-36 and the LIhFE was high. The Kuder-Richardson coefficient in both cases was 0.95 .

\section{CONTENT VALIDITY}

Twenty one patients (35\%) indicated that they were affected by their heart condition in ways that were not addressed by the LVD-36. Five patients described employment related problems. Problems with sexual activity were described by two patients. A lack of concentration was described by two patients. On this point, it is worth noting that an item "I have trouble concentrating" had been included in the original item pool but was removed during the objective item reduction process. $^{89}$ All other comments were unique to the individuals who made them. The majority of patients $(65 \%)$ indicated that there had been no other important ways in which their heart condition affected them. Patients who provided
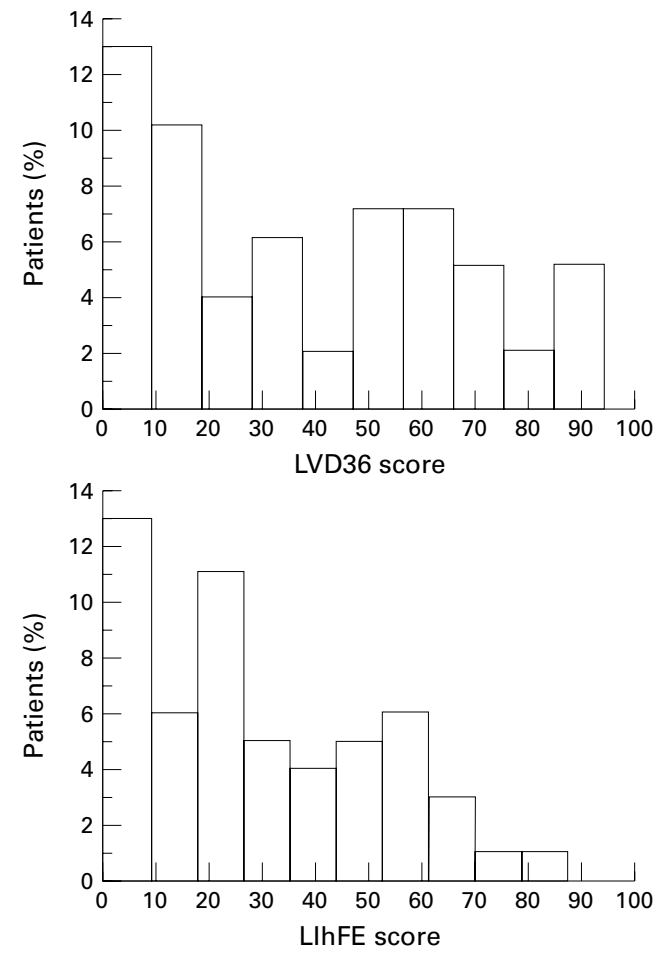

Figure 1 Histogram of LVD-36 and LIhFE scores. 
Table 2 Correlation coefficients (Pearson's $r$ ) between the heart failure questionnaires and the general health measured using the SF-36

\begin{tabular}{lll}
\hline SF-36 components & LVD-36 & LIhFE \\
\hline Physical functioning & $-0.74^{\star \star}$ & $-0.74^{\star \star}$ \\
Role-physical & $-0.68^{\star \star}$ & $-0.67^{\star \star}$ \\
Role-emotional & $-0.52^{\star \star}$ & $-0.48^{\star \star}$ \\
Social functioning & $-0.70^{\star \star}$ & $-0.70^{\star \star}$ \\
Mental health & $-0.46^{\star \star}$ & $-0.41^{\star}$ \\
Vitality & $-0.75^{\star \star}$ & $-0.66^{\star \star}$ \\
Bodily pain & $-0.73^{\star \star}$ & $-0.72^{\star \star}$ \\
General health & $-0.67^{\star \star}$ & $-0.59^{\star \star}$ \\
Mental component score & $-0.48^{\star \star}$ & $-0.43^{\star}$ \\
Physical component score & $-0.75^{\star \star}$ & $-0.73^{\star \star}$ \\
\hline
\end{tabular}

${ }^{\star} \mathrm{p}=0.001 ;{ }^{\star \star} \mathrm{p}=0.0001$.

LihFE, Minnesota living with heart failure questionnaire; LVD36 , left ventricular dysfunction questionnaire (36 items); SF-36, short form 36 item general health questionnaire.

comments were compared with those who did not. Unpaired $t$ tests showed no significant difference between the two groups of patients in terms of age, echocardiographic variables, achieved $\mathrm{VO}_{2} \max$, exercise duration, SF-36 scores, LVD-36 scores, or LIhFE scores ( $t$ ranged from -0.03 to $-1.55 ; \mathrm{p}>0.05) \cdot \chi^{2}$ Tests were used to compare the aetiologies, NYHA class, and drug treatment in the two groups of patients. There were no differences $\left(\chi^{2}\right.$ ranged from 0.09 to $\left.3.39 ; \mathrm{p}>0.05\right)$.

\section{CONCURRENT VALIDITY}

Association with SF-36

The association between the two condition specific questionnaires and the SF-36 was examined using Pearson's correlation coefficient. The results are given in table 2 . The LVD-36 and the LIhFE were similarly correlated with the SF-36, although all correlations were marginally stronger with the LVD-36 than with the LIhFE. Both the LVD-36 and the LIhFE correlated less strongly with SF-36 scores relating to psychological wellbeing than with the scores relating to physical wellbeing.

Association with exercise capacity

The LVD-36 and the LIhFE both correlated significantly with exercise performance (table 3). The correlation coefficients between the LVD-36 and exercise variables were marginally stronger than the corresponding correlations between the LIhFE and exercise performance.

\section{Association with NYHA classification}

The association between LVD-36 and NYHA classification was examined using analysis of variance (ANOVA). The result was significant $(\mathrm{F}=40.6 ; \mathrm{p}<0.0001)$. The ANOVA between

Table 3 Pearson's correlation coefficients between echocardiographic variables, exercise test results, and health status scores

\begin{tabular}{|c|c|c|c|c|}
\hline & \multicolumn{2}{|c|}{ LVD-36 } & \multicolumn{2}{|l|}{ LIhFE } \\
\hline \multicolumn{5}{|l|}{ Exercise test $(n=38)$} \\
\hline Achieved $\mathrm{Vo}_{2}(\mathrm{ml} / \mathrm{min} / \mathrm{kg})$ & \multicolumn{2}{|c|}{$0.52^{\star \star \star}$} & \multicolumn{2}{|c|}{$0.49^{\star \star}$} \\
\hline Exercise duration (s) & \multicolumn{2}{|c|}{$0.45^{\star \star}$} & \multicolumn{2}{|l|}{$0.38^{\star}$} \\
\hline \multicolumn{5}{|l|}{ Echocardiogram $(n=60)$} \\
\hline Left ventricle cavity in diastole $(\mathrm{cm})$ & 0.12 & (NS) & 0.08 & (NS) \\
\hline Left ventricle cavity in systole (cm) & 0.22 & (NS) & 0.17 & (NS) \\
\hline Systolic shortening fraction $(\%)$ & $-0.27^{\star}$ & & -0.22 & (NS) \\
\hline Ejection fraction $(\%)$ & -0.25 & (NS) & -0.22 & (NS) \\
\hline
\end{tabular}

${ }^{\star} \mathrm{p}<0.05 ;{ }^{\star \star} \mathrm{p}<0.01 ;{ }^{\star \star \star} \mathrm{p}<0.001$.

Note: In technically difficult echocardiograms, no value was provided for ejection fraction. For this reason, only 46 of the 60 patients had ejection fraction measured using an echocardiogram.
LIhFE and NYHA was also significant $(\mathrm{F}=31.6 ; \mathrm{p}<0.0001)$. Post hoc tests using Fisher's protected least significance difference revealed that, for both the LVD-36 and the LIhFE, significant differences were found between all NYHA classes, except between classes III and IV.

Association with echocardiogram

Correlations between the LVD-36 and echocardiographic measurements were weak (table 3). Of the four variables used, systolic shortening fraction was the only one to correlate significantly with LVD-36 score $(r=-0.27 ; \mathrm{p}=0.04)$. There was no significant correlation between the LIhFE score and any echocardiographic variable.

\section{RESPONSIVENESS}

Association with global change

The relation between change in questionnaire scores over six months and global change rating was examined using ANOVA. There was a significant association between overall change in health and change in LVD-36 score $(F=5.7$; $\mathrm{p}<0.001)$ or change in LihFE score $(\mathrm{F}=3.7$; $\mathrm{p}<0.01)$. Figure 2 shows a linear trend between global change and change in LVD-36 score; this pattern was not so clear with the LihFE. Using this questionnaire, patients who rated themselves as "a little better" had a mean deterioration in LIhFE score of $8.3 \%$, while patients who rated themselves as "about the same" had a mean improvement in LIhFE score of $3.8 \%$. Relative to the mean change in score, the $95 \%$ confidence intervals around the LVD-36 scores were tighter than those for the LihFE. The change in LVD-36 score for the patients who were "very much better" and "a little worse" were significantly different from zero. In contrast, none of the changes in the LihFE questionnaire achieved significance. This suggests that the LVD-36 may provide a more precise and sensitive estimate of changes in health status than the existing questionnaire.

\section{Effect size}

Patients were grouped according to their rating of global change. The effect size for each of the two questionnaires was calculated for each group. The results are presented in table 4 . Cohen has defined an effect size of 0.20 as small, one of 0.50 as moderate, and one of 0.80 or greater as large. ${ }^{12}$ The effect sizes of the LVD-36 for each global change group were matched in magnitude to Cohen's definition. The effect size was large for patients rating themselves "very much better"; moderate for those rating themselves "much better" and "much worse"; and small for those rating themselves "a little better" and "a little worse". It will be noted that in patients who rated themselves "about the same", the effect size was effectively zero.

The effect sizes for the LIhFE in patients rating themselves as "very much better", "much better", and "a little worse" were similar to the corresponding values calculated for the LVD-36. However, the LIhFE effect size for patients rating themselves as a "little better" 

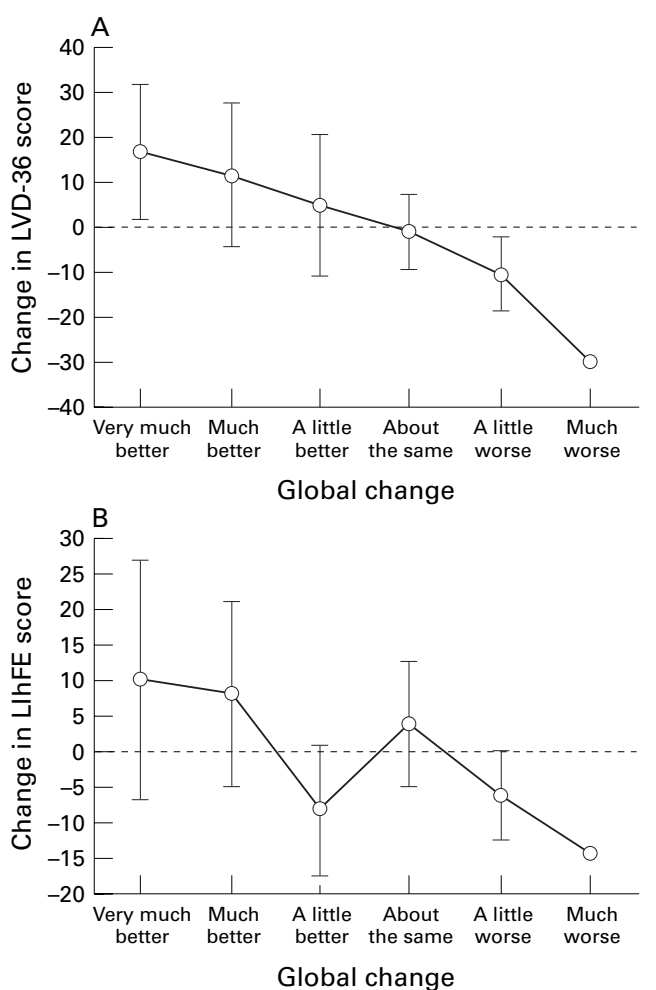

Figure 2 Association between the patients'score for global change and change in scores for (A) LVD-36 and (B) LIhFE over six months. Error bars show $95 \%$ confidence intervals.

showed a moderate deterioration in health status. Furthermore, in patients who rated themselves "about the same", the effect size for the LIhFE suggested a small improvement.

\section{Discussion}

These results suggest that the LVD-36 is suitable for measuring health status in patients with left ventricular dysfunction. It is short and simple to complete and, for the majority of individuals, covers all areas of life that are impaired because of their disease. It shows high levels of repeatability and internal consistency. Its scores were significantly associated with a range of established measures of disease severity, except echocardiographic variables. The absence of a correlation with echocardiographic function is unsurprising. While impaired left ventricular function is the underlying pathophysiological problem in left ventricular dysfunction, its effects are mediated by numerous pathways. These include different sensations such as breathlessness and fatigue and secondary processes in skeletal muscles including biochemical and physiological effects, and disuse atrophy. It will be noted that health status was clearly associated with both peak exercise performance and exercise endurance. In all tests of validity, the LVD-36 performed similarly or marginally better than the LIhFE.

Changes in LVD-36 score were strongly related to the patients' perception of their overall change. In contrast, the relation between the patients' perception of their overall change and change in the LIhFE score was
Table 4 Effect sizes calculated for the change in LVD-36 and LIhFE scores over six months, categorised by the patients' estimate of the overall change in their state

\begin{tabular}{lcr}
\hline & LVD-36 & LIhFE \\
\hline Very much better & 0.99 & 0.92 \\
Much better & 0.46 & 0.45 \\
A little better & 0.25 & -0.44 \\
About the same & -0.03 & 0.20 \\
A little worse & -0.26 & -0.28 \\
\hline
\end{tabular}

Note: Only one patient was "Much worse", so no effect size was calculated.

not as strong. Patients rating themselves as "a little better" had a mean decrease of $8.3 \%$ (that is, worse) using the LIhFE, in comparison to an increase of $4.8 \%$ (that is, better) using the LVD-36. Unlike the LIhFE, the effect sizes for the LVD-36 followed the rank order of the global rating of change. These results suggests that the LVD-36 may be a more reliable measure of changes in health than the LIhFE. In this context it should be appreciated that this study measured spontaneous changes in the health of patients with left ventricular dysfunction. Further studies are required to test its sensitivity to therapeutic intervention. We found that responses to the questionnaire were not affected by the aetiology of the patient's heart disease. In part this would have been the result of the item selection process-in eliminating items that were related to age, sex, and disease duration. ${ }^{8}{ }^{9}$ We were unable to recruit many patients in some aetiological groups despite recruiting the subjects from a tertiary referral clinic. Further work would be required to confirm our observation that the aetiology of left ventricular dysfunction does not have any effect on responses to the questionnaire, but this would require a large study. Such a study should also include patients in NYHA class V.

\section{CONCLUSIONS}

The LVD-36 has shown a high level of reliability and validity, and is responsive to changes in health. It is applicable to all aetiological groups, including patients with dilated cardiomyopathy who were apparently asymptomatic in terms of the absence of oedema or clinical breathlessness. It is short, simple to complete, and the wording is clear and unambiguous. It may be administered in the clinic or over the telephone, and it is easy to score. It has been has suggested that a health status measure should be: "appropriate for the study population, . . reliable, valid, able to discriminate between different groups, easy to administer, uncomplicated to analyse, and preferably suitable for completion by the patients themselves." ${ }^{13}$ Results from this study suggest that the LVD-36 meets these requirements for measuring health status in patients with left ventricular dysfunction.

This study was supported by a grant from the British Heart Foundation. We than Professor W A McKenna and Professor K B Saunders for allowing us to study patients under their care.

1 Guyatt GH, Nogradi S, Halcrow S, et al. Development and testing of a new measure of health status for clinical trials in heart failure. $\mathcal{F}$ Gen Intern Med 1989;4:101-7. 
2 Rector TS, Kubo SH, Cohn JN. Patients's self-assessment of their congestive heart failure. Part 2. Content, reliability and validity of a new measure, the Minnesota Living with Heart Failure questionnaire. Heart Failure 1993;3:198209

3 Wiklund I, Lindvall KAJ, Swedberg K, et al. Self-assessment of quality of life in severe heart failure. Scand $\mathcal{F}$ Psycho $1987 ; 28: 220-5$.

Wiklund I, Karlberg J. Evaluation of quality of life in clinica trials. Control Clin Trials 1991;12:204-16S.

5 Rector TS, Johnson G, Rector, et al. Evaluation by patients with heart failure of the effects of enalapril compared with hydralazine plus isosorbide dinitrate on quality of life. Circulation 1993;87(suppl VI):71-7.

6 Quirk FH. Quality of life in respiratory disease. London: University of London, 1996. [PhD thesis.]

7 Quirk FH, Jones PW. Back to basics: how many items can adequately represent health-related quality of life in airways disease. Eur Respir Rev 1997;7:50-2.
8 O'Leary CJ, Jones PW. The influence of decisions made by developers on health status questionnaire content. Qual Life Res 1998;7:545-50.

9 O'Leary CJ. Quantifying the impact of left ventricular dysfunction on daily life and well being. London: University of London, 1998. [PhD thesis.]

10 Gardin JM, Henry WL, Savage DD, et al. Echocardiographic measurements in normal subjects: evaluation of an adult population without clinically apparent heart disease. adult population without clinicall

11 Goldman L, Hashmito B, Cook E, et al. Comparative reproducibility and validity of systems for assessing cardiovascular functional class: advantages of a new specific activity scale. Circulation 1981;64:1227-34.

12 Cohen J. Statistical power analysis for the behavioral sciences. New York: Academic Press, 1977.

13 Hawthorne J. Measuring quality of life. Eur $\mathcal{F}$ Cancer Care 1993;2:77-81.

\section{IMAGES IN CARDIOLOGY}

\section{Cullen's sign after coronary angiography}

A 59 year old woman weighing $60 \mathrm{~kg}$ developed an inferior myocardial infarction and was treated with a combination of intravenous tissue plasminogen activator (tPA) $(15 \mathrm{mg}$ bolus and $35 \mathrm{mg}$ infusion over one hour), abciximab $(0.25 \mathrm{mg} / \mathrm{kg}$ bolus and $0.125 \mu \mathrm{g} / \mathrm{kg} /$

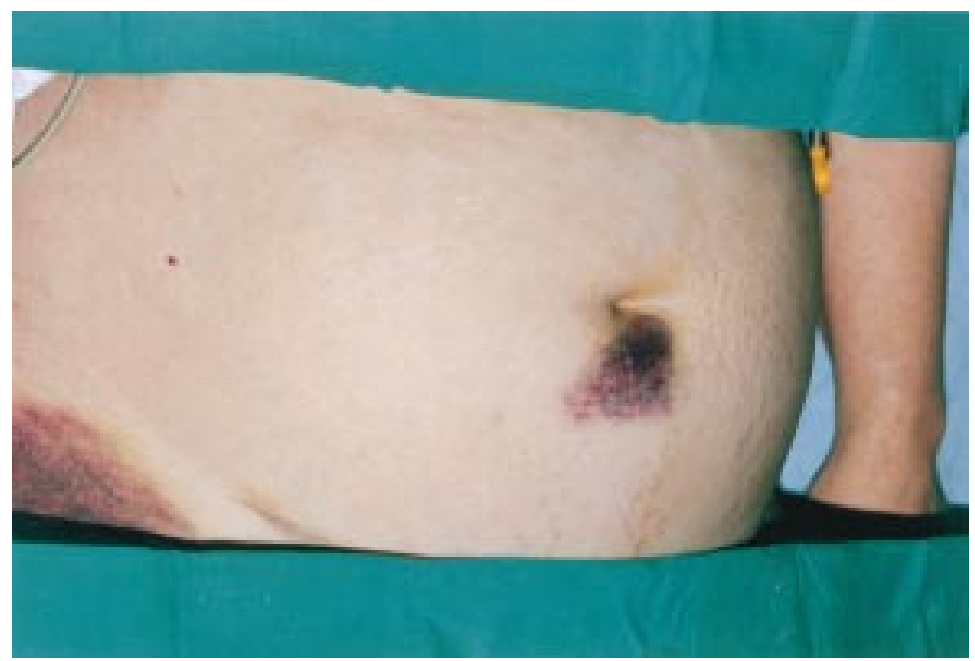

min infusion), and heparin (60 units/kg bolus and 15 units/kg/hour infusion). Coronary arteriography was carried out one hour after initiation of treatment. Right femoral artery cannulation was not successful and the procedure was performed using the left femoral approach. The patient developed an extensive haematoma at the right groin site, which extended into the retroperitoneal space and required blood transfusion.

Twelve hours later Cullen's sign was noted. No subcutaneous heparin had been administered. The peri-umbilical bruising gradually faded over the next few days. The right groin haematoma resolved without any sequelae.

Cullen's sign is the result of blood tracking along the falciform ligament from the retroperitoneal space. It is named after TS Cullen, professor of gynaecology at Johns Hopkins University, who described the sign in 1919 in a patient with a ruptured ectopic pregnancy. It is more commonly observed in acute pancreatitis and in rupture of an abdominal aortic aneurysm.

MS SPENCE SW WEBB 